\title{
A pilot study of a group mindfulness-based cognitive-behavioral intervention for smartphone addiction among university students
}

\author{
YUKUN LAN ${ }^{1}$, JIAO-ER DING ${ }^{1}$, WEI LI ${ }^{2}$, JIANG LI ${ }^{1,3,4,5 *}$, YIFEI ZHANG ${ }^{1}$, MINGBO LIU ${ }^{2}$ and HUA FU ${ }^{1,3,4}$ \\ ${ }^{1}$ Department of Preventive Medicine, School of Public Health, Fudan University, Shanghai, China \\ ${ }^{2}$ Institute of Psychological Health Education, Fudan University, Shanghai, China \\ ${ }^{3}$ Health Communication Institute, Fudan University, Shanghai, China \\ ${ }^{4}$ The Key Laboratory of Public Health Safety, Fudan University, Shanghai, China \\ ${ }^{5}$ Fudan-Pudong Preventive Medicine Institute, Fudan University, Shanghai, China
}

(Received: May 26, 2018; revised manuscript received: August 21, 2018; second revised manuscript received: September 8, 2018; accepted: September 9, 2018)

\begin{abstract}
Background and aims: Mindfulness-based intervention (MBI) has been applied in behavioral addiction studies in recent years. However, few empirical studies using MBI have been conducted for smartphone addiction, which is prevalent among Chinese university students. The aim of this study was to investigate the effectiveness of a group mindfulness-based cognitive-behavioral intervention (GMCI) on smartphone addiction in a sample of Chinese university students. Methods: Students with smartphone addiction were divided into a control group $(n=29)$ and an intervention group $(n=41)$. The students in the intervention group received an 8-week GMCI. Smartphone addiction was evaluated using scores from the Mobile Phone Internet Addiction Scale (MPIAS) and self-reported smartphone use time, which were measured at the baseline (1st week, T1), post-intervention (8th week, T2), the first follow-up (14th week, T3), and the second follow-up (20th week, T4). Results: Twenty-seven students in each group completed the intervention and the follow-up. Smartphone use time and MPIAS scores significantly decreased from T1 to T3 in the intervention group. Compared with the control group, the intervention group had significantly less smartphone use time at T2, T3, and T4 and significantly lower MPIAS scores at T3. Discussion and conclusion: This pilot study demonstrated that the GMCI could significantly alleviate smartphone addiction among university students.
\end{abstract}

Keywords: mindfulness-based intervention, cognitive-behavioral therapy, group intervention, smartphone addiction, university students

\section{INTRODUCTION}

Smartphones are one of the most popular electronic products in the world today. They provide substantial convenience, but smartphone addiction is becoming a serious problem and is increasingly prevalent worldwide (Ding \& Li, 2017). According to data from recent surveys, the rate of problematic smartphone usage is estimated at $21.3 \%$ among students in China (Long et al., 2016), and 10\%-25\% of American people tend to have problematic cell phone usage (Smetaniuk, 2014). A cross-sectional study conducted in the UK found that $10 \%$ of students exhibited problematic mobile phone usage (Lopez-Fernandez, Honrubia-Serrano, Freixa-Blanxart, \& Gibson, 2014), whereas a study in Switzerland reported that $16.9 \%$ of students had a smartphone addiction problem (Haug et al., 2015). A meta-analysis showed that the prevalence of smartphone addiction in India ranges from 39\% to $44 \%$ among adolescents (Davey \& Davey, 2014).

Smartphone addiction can lead to ill health, including physical, psychological, and social issues (Ding \& Li, 2017). It is generally considered to be a mental health concern and, more specifically, a type of behavioral addiction
(Griffiths, 2000; Lin et al., 2016; Young, 1999). According to a literature review, common treatment options for behavioral addiction include cognitive-behavioral therapy (CBT), motivational intervention, and mindfulness behavioral cognitive treatment, which can be conducted separately or jointly (Kim, 2013; Shonin, Van Gordon, \& Griffiths, 2014a). The principle of these interventional approaches focuses on the stimulation of personal cognition and behavior and changing feelings and thoughts.

Mindfulness derives from Buddhist meditation and emphasizes the engagement of full, direct, and active awareness of experienced phenomena that is spiritual and is maintained from one moment to the next (Shonin, Van Gordon, \& Griffiths, 2013; Shonin et al., 2014a). Through mindfulness techniques, participants learn to increase their perceptual distance from mental urges. This approach has

* Corresponding author: Jiang Li; Department of Preventive Medicine, School of Public Health, Fudan University, Yixueyuan Road 138, PO Box 248, Shanghai 200032, China; Phone: +86 15 7215 64581; Fax: +86 215423 7509; E-mail: Lijiang_fd@fudan. edu.cn

This is an open-access article distributed under the terms of the Creative Commons Attribution-NonCommercial 4.0 International License, which permits unrestricted use, distribution, and reproduction in any medium for non-commercial purposes, provided the original author and source are credited, a link to the CC License is provided, and changes - if any - are indicated. 
been deemed suitable for treating behavioral addictions for the following reasons: (a) meditation can reduce relapse and withdrawal symptoms, (b) mindfulness can regulate an addiction-related distressed emotional state, (c) the techniques can help in recognizing the intrinsic value of life instead of the superficial reward of addictive activities, (d) salience can be reduced, and (e) patience can be improved (Van Gordon et al., 2017).

In recent years, people have applied mindfulness approaches in the treatment of various mental disorders, including behavioral addiction (Luberto, Magidson, \& Blashill, 2017; Manicavasgar, Parker, \& Perich, 2011; Shonin et al., 2013). One of the most frequently studied areas is the mindfulness-based treatment of pathological gambling (Lisle, Dowling, \& Allen, 2012). This type of approach has also been applied to treat workaholism (Shonin, Van Gordon, \& Griffiths, 2014b; Van Gordon et al., 2017) and sex addiction (Van Gordon, Shonin, \& Griffiths, 2016).

Some scholars have discussed the feasibility and affirmed the effect of mindfulness-based intervention (MBI) on Internet addiction (Kim, 2013; Shonin et al., 2013). Some studies have even revealed the mechanisms of this type of intervention by quantitatively measuring mindfulness and analyzing its relationship with Internet addiction (Calvete, Gámez-Guadix, \& Cortazar, 2017; Gámez-Guadix \& Calvete, 2016). However, few empirical MBI studies currently exist, especially regarding smartphone addiction (Li, Niu, \& Mei, 2017). The aim of this study was to conduct a pilot program to assess the intervention effect of smartphone addiction based on the process of mindfulness cognitive-behavioral therapy.

\section{METHODS}

\section{Participants}

We applied stratified cluster sampling to select three to six classes from the medical college, the arts college, and the college of science and engineering of a university in Shanghai. Altogether, we distributed 1,091 questionnaires to the students, and 1,044 completed questionnaires $(95.7 \%$ response) were ultimately returned. The average age of the students was $21.3 \pm 1.3$ years, and males accounted for $47.6 \%$ of the sample.

\section{Procedures}

We recruited 70 volunteers from students evaluated as smartphone addicts. Smartphone addiction was determined by a cut-off score $\geq 65$ and self-reported smartphone use time $\geq 2 \mathrm{hr}$ /day. The score was based on the Mobile Phone Internet Addiction Scale (MPIAS), which was developed in our previous study (Hu, Xu, Ding, \& Li, 2017). The MPIAS is a 32-item self-report scale assessing smartphone addiction among college students. The MPIAS items are rated on a 5-point Likert scale, with a total score of 160 points. Forty-one students were assigned to the intervention group, because their schedules matched our arrangement, and the remaining 29 students were assigned to the control group, because they were not confident that they could complete the program. Due to ethical considerations, we gave all participants (both the intervention and the control groups) an educational lecture on smartphone addiction prevention and distributed flyers before the launch of the intervention. Then, 41 students in the intervention group were further divided into five groups according to their schedules. The intervention was implemented in groups. Due to time commitments, 27 of the 41 students in the intervention group and 27 of the 29 students in the control group completed the study.

\section{Program description}

The manual for the group mindfulness-based cognitivebehavioral intervention (GMCI) was developed with precision based on the theoretical framework of group CBT, previous intervention practices, and empirical studies $(\mathrm{Du}$, Jiang, \& Vance, 2010; Segal, Williams, \& Teasdale, 2002). The intervention program consisted of eight sessions, which were administered for each intervention group. There was one session once a week, with each session lasting approximately $1 \mathrm{hr}$. In the first three sessions, the interventions were aimed at cognitive reconstruction. They were as follows: the first session consisted of an orientation and individual feedback on smartphone use incentives; the second session focused on identifying high-risk situations; and the third session focused on identifying negative thoughts and cognition reconstruction. We integrated mindfulness meditation into the intervention under the framework of CBT in the last five sessions: the fourth session taught meditation learning and relaxation training; the fifth session taught participants to cope with relapse; the sixth session focused on other activities to replace smartphone use; the seventh session discussed setting life goals and rules; and the eighth session was spent reviewing the program. The participants were asked to do homework, which included reviewing the contents of the last session and/or practicing mindfulness meditation every day.

\section{Measures}

The assessments were completed at baseline (1st week, T1), post-intervention (8th week, T2), the first follow-up (14th week, T3), and the second follow-up (20th week, T4) for all participants. The details of the intervention process are shown in Figure 1.

\section{Statistical analyses}

The data analyses were performed using SPSS 20.0 for Windows (IBM, Armonk, NY, USA). Descriptive statistics were calculated to examine the participants' demographic characteristics. Repeated-measures analysis of variance (RM-ANOVA) was applied to examine the overall effectiveness of the intervention. Partial $\eta_{\mathrm{p}}{ }^{2}$ provided by RM-ANOVA was used to describe the size effects. Independent-samples $t$-tests were used to compare MPIAS scores and smartphone use time between the groups at T1, T2, T3, and T4. Pairedsamples $t$-tests with Bonferroni correction were conducted for each group to analyze the differences for all intervening variables at $\mathrm{T} 1, \mathrm{~T} 2, \mathrm{~T} 3$, and $\mathrm{T} 4$. 


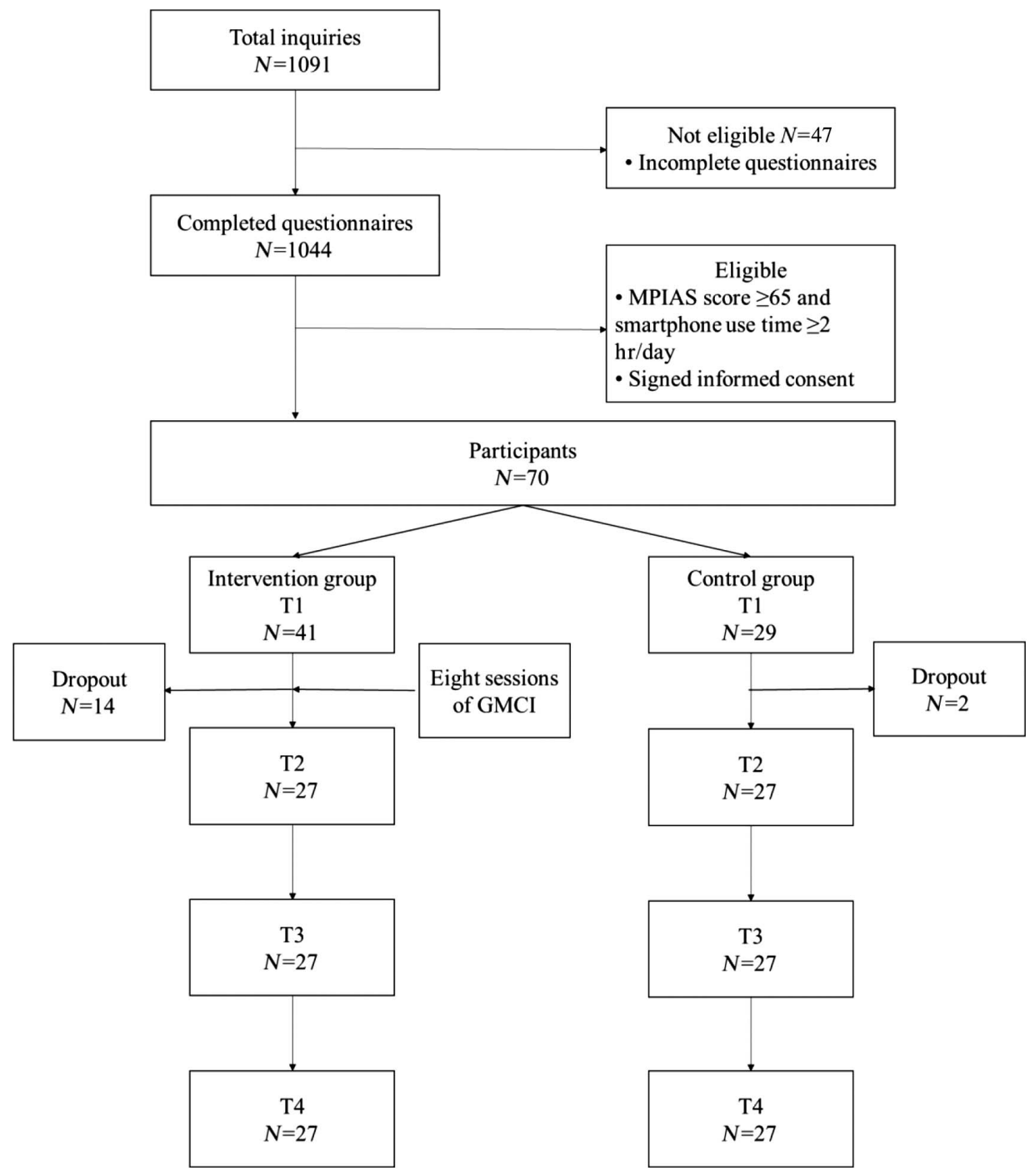

Figure 1. Participant flow. Note. T1 refers to the baseline measurement (1st week), T2 refers to the post-intervention (8th week), T3 is the first follow-up (14th week), and T4 is the second follow-up (20th week). MPIAS: Mobile Phone Internet Addiction Scale; GMCI: group mindfulness-based cognitive-behavioral intervention

\section{Ethics}

The study was approved by the institutional review board of the School of Public Health of Fudan University. All subjects were informed about the study and all provided informed consents.

\section{RESULTS}

There were no statistically significant differences between the intervention group and the control group for age and gender distribution (age: $21.1 \pm 1.7$ vs. $21.2 \pm 1.6$ years, $p=.87$, ANOVA; male/female: $12 / 15$ vs. $10 / 17, p=.58, \chi^{2}$ test). In addition, there were no differences in smartphone use time $\left(t_{52}=-0.912, p=.366\right)$ and MPIAS score $\left(t_{52}=\right.$ $-0.399, p=.691)$ between the two groups at T1.

The results of the RM-ANOVA showed that the interaction effect of Time $\times$ Group was not significant for smartphone use time $[F(3,156)=1.669, p=.213]$ or MPIAS score $[F(3,50)=1.012, p=.395])$, indicating that the effects of the time factor were not significant between the groups. The time effects were significant for both smartphone use time $[F(3,156)=7.242, p<.001]$ and MPIAS score $[F(3,50)=9.382, p<.001]$, and the group effect was significant for smartphone use time $[F(1,52)=7.242$, $p=.005]$. The $\eta_{\mathrm{p}}{ }^{2}$ values of time, group, and Time $\times$ Group effects were $0.122,0.144$, and 0.028 for smartphone use time and the values for the MPIAS score were $0.234,0.038$, and 0.022 , respectively. The results of the independentsamples $t$-test revealed significant differences between the two groups for smartphone use time at T2 $\left(t_{39}=-3.239\right.$, $p=.002)$, T3 $\left(t_{52}=-2.424, p=.019\right)$, and $\mathrm{T} 4 \quad\left(t_{52}=\right.$ $-2.819, p=.007)$ and for the MPIAS score at T3 $\left(t_{52}=\right.$ $-2.368, p=.022$ ). Moreover, for the intervention group, paired-samples $t$-tests found that smartphone use times at T2 $\left(t_{26}=3.623, p=.001\right), \mathrm{T} 3\left(t_{26}=6.4, p<.001\right)$, and T4 $\left(t_{26}=3.017, p=.006\right)$ were significantly less than smartphone use time at T1 and that MPIAS scores were not only significantly lower at T3 $\left(t_{26}=4.472, p<.001\right)$ and T4 $\left(t_{26}=3.967, p=.001\right)$ compared with $\mathrm{T} 1$ but were also significantly lower at T3 $\left(t_{26}=3.502, p=.002\right)$ and $\mathrm{T} 4$ $\left(t_{26}=3.032, p=.005\right)$ compared with T2. In the control group, smartphone use times were not significantly different 


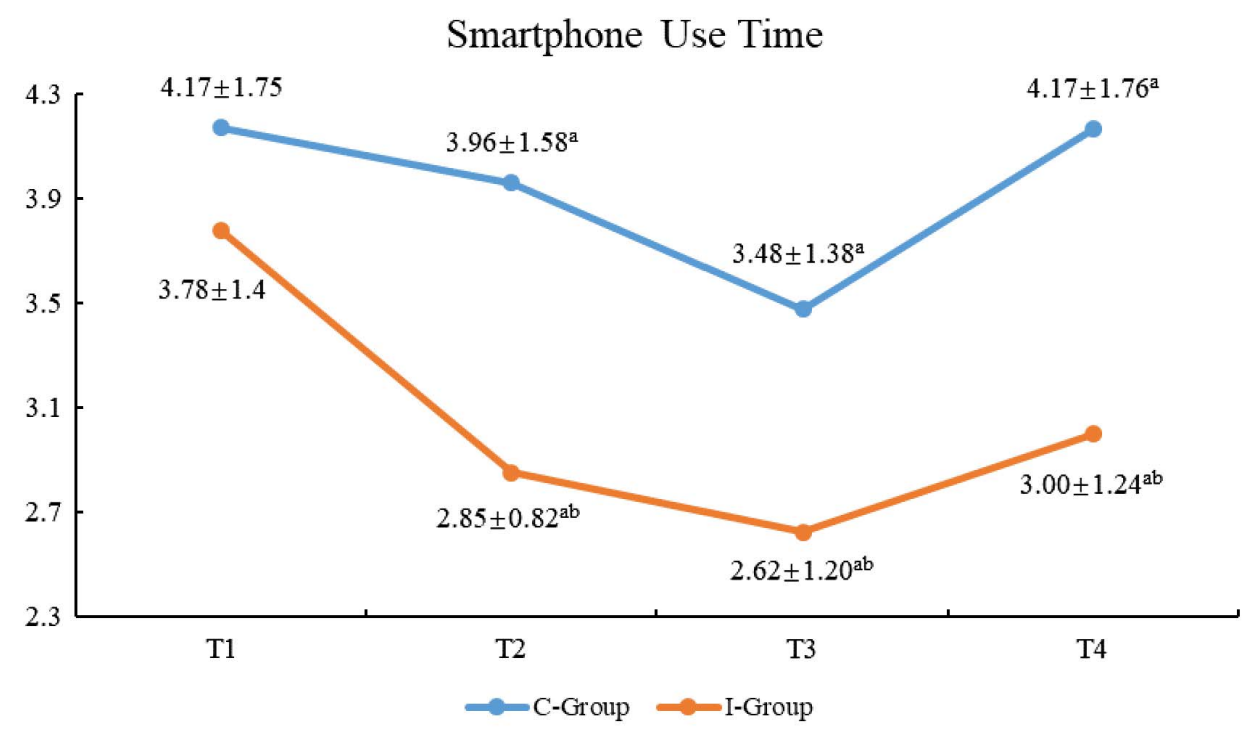

MPIAS Score

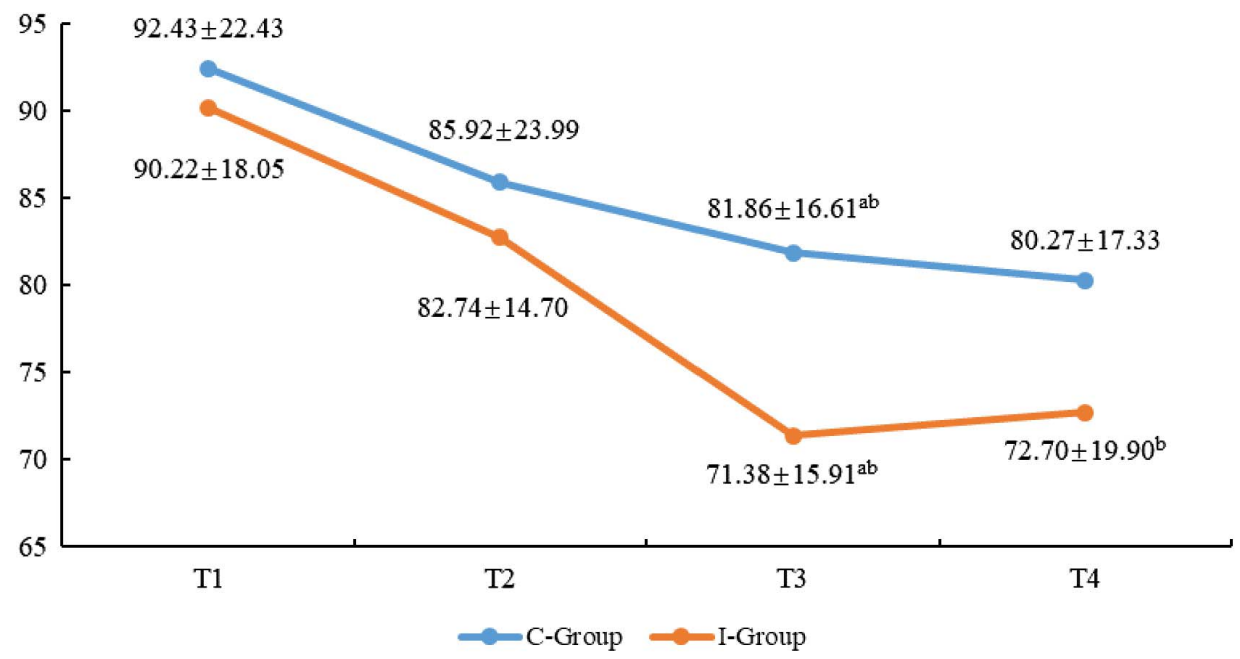

Figure 2. The changes in the estimated marginal means for the four time points according to the intervention and control groups. Note. The figure illustrates the intervention's effects on smartphone addiction. MPIAS: Mobile Phone Internet Addiction Scale; I-group: intervention group; C-group: control group; T1: baseline (1st week); T2: post-intervention (8th week); T3: the first follow-up (14th week); T4: the second follow-up (20th week); the numbers are shown as the mean \pm standard deviation. Values with superscript "a" indicate that the means for the I- and C-groups at the same time point are significantly different; "b" indicates that the mean for time point T2, T3, or T4 is significantly smaller than the mean value for $\mathrm{T} 1$ in the I- or C-group

according to the time variable, while the MPIAS score at T3 $\left(t_{26}=2.994, p=.006\right)$ was significantly lower than that at T1. Figure 2 shows the features of smartphone addiction at each measured time point for both groups.

\section{DISCUSSION AND CONCLUSIONS}

In many studies, MBIs have achieved satisfactory effects on some behavioral addictions, including pathological gambling, workaholism, sex addiction, and Internet addiction (Lisle et al., 2012; Shonin et al., 2013, 2014b; Son, 2011; Van Gordon et al., 2016, 2017). However, limited MBI studies have been conducted on smartphone addiction prevention. We discovered only two relevant studies published in Chinese: a case study found that mindfulness therapy could effectively improve smartphone addiction, impulsivity, and anxiety among medical students (Li et al., 2017), and another study demonstrated that mindfulness-based cognitive therapy could significantly decrease uncontrolled response, withdrawal, and inefficiency regarding smartphone addiction among college students (Zhang \& Zhu, 2014).

The key treatment mechanisms of mindfulness include two aspects. One is a perceptual shift in the mode of responding and relating to sensory and cognitive-affective stimuli that permit individuals to objectify their cognitive processes and to apprehend them as passing phenomena. The other is a reduction in relapse and withdrawal symptoms by replacing maladaptive addictive behaviors with mindfulness (Shonin et al., 2013). In this study, the key content in the first 3-week intervention involved constructing correct cognition of smartphone use by clarifying the root purpose of smartphone use, the behavior itself, and the consequences. Cognition reconstruction is 
based on mindfulness therapy. The participants were subsequently asked to objectify their behavior and dissociate the affection related to smartphones in the meditation. From the fifth to the seventh sessions, the participants were trained to deal with relapse. Mindfulness teaching can help students reduce their desire for smartphone use and relieve their discomfort when they have to leave their smartphone. Moreover, the participants were asked to perform mindfulness practice every day during the program, which also exercised their persistence, as reflected in this study results. Six weeks after the intervention program, both the smartphone use time and the MPIAS score decreased consistently (Figure 2, T3 vs. T2). During the first follow-up survey, more than half of the participants (14/27) in the intervention group noted that they had continued practicing the mindfulness exercise every day.

The advantage of this GMCI is that it is structuralized and programmed. Accordingly, the GMCI could be easily conducted by an instructor who has received only short-term training (which is the method applied in this study). Since the effects of the time factor did not differ between the groups, the significant differences in smartphone use time and MPIAS scores between the intervention group and the control group demonstrate that the GMCI can relieve smartphone addiction. Furthermore, the effect of the intervention was sustained from post-intervention (T2) to the second follow-up (T4).

However, final examinations and the beginning of summer vacation occurred during the intervention, which might have affected the results of the study. For example, from T2 to T3, the students had to prepare for their final examinations, which reduced their smartphone use time. In addition, at T4, summer vacation had begun, offering students more time to engage in outdoor activities. This could also have alleviated smartphone addiction. Therefore, both smartphone use time and MPIAS scores at T2 and T3 decreased not only for the intervention group but also for the control group when compared with T1, which occurred during an early stage of the semester. In addition, smartphone use time increased at T4 compared with T3 for both groups, but the MPIAS score decreased at T4 compared with T3 only for the control group. Another limitation of this study is that we did not control the confounders, such as the participants' activity level, satisfaction, compliance in the program, and other factors. Due to our limited budget, we did not measure some of these variables. Furthermore, because of the small sample size, we could not conduct a stratified analysis. In addition, 14 of 41 students in the intervention group dropped out of the program, which might lead to information bias and affect the study results.

In conclusion, the pilot study demonstrated the effectiveness of the GMCI on smartphone addiction. A further study with a multicenter, randomized controlled design will be conducted in heterogeneous populations to validate the results.

Funding sources: This study was supported by a grant from the construction of the key disciplines of the fourth round of the public health 3-year action plan of the Shanghai Health and Family Planning Commission under award number 15GWZK1001.
Authors' contribution: YL co-conducted the study, performed the statistical analysis, and wrote the manuscript. J-ED and WL co-designed and co-conducted the study. YZ co-conducted the study. JL was involved in the entire study process. ML and HF supplied study supervision. All the authors had full access to all the data in the study and take responsibility for the integrity of the data and the accuracy of the data analysis. YL, J-ED, WI, and JL contributed equally to this work.

Conflict of interest: The authors declare no conflict of interest.

Acknowledgements: The authors would like to thank all of the participants in the study.

\section{REFERENCES}

Calvete, E., Gámez-Guadix, M., \& Cortazar, N. (2017). Mindfulness facets and problematic Internet use: A six-month longitudinal study. Addictive Behaviors, 72, 57-63. doi:10.1016/j. addbeh.2017.03.018

Davey, S., \& Davey, A. (2014). Assessment of smartphone addiction in Indian adolescents: A mixed method study by systematicreview and meta-analysis approach. International Journal of Preventive Medicine, 5(12), 1500-1511. Retrieved from http:// www.ijpm.mui.ac.ir/index.php/ijpm/article/view/1459/0

Ding, D., \& Li, J. (2017). Smartphone overuse - A growing public health issue. Journal of Psychology \& Psychotherapy, 7(1), 289. doi:10.4172/2161-0487.1000289

Du, Y. S., Jiang, W. Q., \& Vance, A. (2010). Longer term effect of randomized, controlled group cognitive behavioural therapy for Internet addiction in adolescent students in Shanghai. Australian and New Zealand Journal of Psychiatry, 44(2), 129-134. doi:10.3109/00048670903282725

Gámez-Guadix, M., \& Calvete, E. (2016). Assessing the relationship between mindful awareness and problematic Internet use among adolescents. Mindfulness, 7(6), 1281-1288. doi:10.1007/ s12671-016-0566-0

Griffiths, M. (2000). Does Internet and computer "addiction" exist? Some case study evidence. CyberPsychology \& Behavior, 3(2), 211-218. doi:10.1089/109493100316067

Haug, S., Castro, R. P., Kwon, M., Filler, A., Kowatsch, T., \& Schaub, M. P. (2015). Smartphone use and smartphone addiction among young people in Switzerland. Journal of Behavioral Addictions, 4(4), 299-307. doi:10.1556/2006.4.2015.037

Hu, D. D., Xu, Y., Ding, J. E., \& Li, J. (2017). Development of Mobile Phone Internet Addiction Scale for college students. Chinese Journal of Health Education, 33(6), 505-508. doi:10.16168/j.cnki.issn.1002-9982.2017.06.006

Kim, H. (2013). Exercise rehabilitation for smartphone addiction. Journal of Exercise Rehabilitation, 9(6), 500-505. doi:10. 12965/jer.130080

Li, L., Niu, Z. M., \& Mei, S. L. (2017). The mindfulness cognitivebehavioral group therapy of medical student'smartphone addiction in group counseling course. China Higher Medical Education, 5, 37-38. doi:10.3969/j.issn.1002-1701.2017.05.016

Lin, Y. H., Chiang, C. L., Lin, P. H., Chang, L. R., Ko, C. H., Lee, Y. H., \& Lin, S. H. (2016). Proposed diagnostic criteria for 
smartphone addiction. PLoS One, 11(11), e0163010. doi:10.1371/journal.pone. 0163010

Lisle, S. M. D., Dowling, N. A., \& Allen, J. S. (2012). Mindfulness and problem gambling: A review of the literature. Journal of Gambling Behavior, 28(4), 719-739. doi:10.1007/s10899011-9284-7

Long, J., Liu, T. Q., Liao, Y. H., Qi, C., He, H. Y., Chen, S. B., \& Billieux, J. (2016). Prevalence and correlates of problematic smartphone use in a large random sample of Chinese undergraduates. BMC Psychiatry, 16(1), 408. doi:10.1186/s12888016-1083-3

Lopez-Fernandez, O., Honrubia-Serrano, L., Freixa-Blanxart, M., \& Gibson, W. (2014). Prevalence of problematic mobile phone use in British adolescents. Cyberpsychology, Behavior and Social Networking, 17(2), 91-98. doi:10.1089/cyber. 2012.0260

Luberto, C. M., Magidson, J. F., \& Blashill, A. J. (2017). A case study of individually delivered mindfulness-based cognitive behavioral therapy for severe health anxiety. Cognitive and Behavioral Practice, 24(4), 484-495. doi:10.1016/j.cbpra. 2016.10.001

Manicavasgar, V., Parker, G., \& Perich, T. (2011). Mindfulnessbased cognitive therapy vs cognitive behaviour therapy as a treatment for non-melancholic depression. Journal of Affective Disorders, 130(1-2), 138-144. doi:10.1016/j.jad.2010.09.027

Segal, Z. V., Williams, J. M. G., \& Teasdale, J. D. (2002). Mindfulness-based cognitive therapy for depression: A new approach to preventing relapse. New York, NY: Guilford Press.

Shonin, E., Van Gordon, W., \& Griffiths, M. D. (2013). Mindfulness as a treatment for behavioural addiction. Journal of Addiction Research \& Therapy, 5(1), e122. doi:10.4172/ 2155-6105.1000e122
Shonin, E., Van Gordon, W., \& Griffiths, M. D. (2014a). Are there risks associated with using mindfulness for the treatment of psychopathology? Clinical Practice, 11(4), 389-392. doi: $10.2217 /$ cpr.14.23

Shonin, E., Van Gordon, W., \& Griffiths, M. D. (2014b). The treatment of workaholism with meditation awareness training: A case study. Explore (NY), 10(3), 193-195. doi:10.1016/j. explore.2014.02.004

Smetaniuk, P. (2014). A preliminary investigation into the prevalence and prediction of problematic cell phone use. Journal of Behavioral Addictions, 3(1), 41-53. doi:10.1556/JBA.3.2014.004

Son, C. (2011). Effects of mindfulness-based cognitive therapy on Internet addiction level, anxiety, and stress in college students with Internet addiction. Korean Journal of Clinical Psychology, 30(4), 825-843. doi:10.15842/kjcp.2011.30.4.001

Van Gordon, W., Shonin, E., Dunn, T. J., Garcia-Campayo, J., Demarzo, M. M. P., \& Griffiths, M. D. (2017). Meditation awareness training for the treatment of workaholism: A controlled trial. Journal of Behavioral Addictions, 6(2), 212-220. doi:10.1556/2006.6.2017.021

Van Gordon, W., Shonin, E., \& Griffiths, M. D. (2016). Meditation awareness training for the treatment of sex addiction: A case study. Journal of Behavioral Addictions, 5(2), 363-372. doi:10.1556/2006.5.2016.034

Young, K. S. (1999). Internet addiction: Symptoms, evaluation and treatment. In L. VandeCreek \& T. L. Jackson (Eds.), Innovations in clinical practice: A source book (Vol. 17, pp. 19-31). Sarasota, FL: Professional Resource Press. Retrieved from http://www.netaddiction.com/articles/symptoms.pdf.

Zhang, X. X., \& Zhu, H. X. (2014). The intervention effect of mindfulness-based cognitive therapy on college students with mobile phone addiction. Studies of Psychology and Behavior, 12(3), 391-394. doi:10.3969/j.issn.1672-0628.2014.03.019 